\title{
Algorithms for Quantum Control without Discontinuities; Application to the Simultaneous Control of two Qubits
}

\author{
Domenico D'Alessandro and Benjamin Sheller \\ Department of Mathematics, Iowa State University \\ April 22, 2019
}

\begin{abstract}
We propose a technique to design control algorithms for a class of finite dimensional quantum systems so that the control law does not present discontinuities. The class of models considered admits a group of symmetries which allows us to reduce the problem of control to a quotient space where the control system is 'fully actuated'. As a result we can prescribe a desired trajectory which is, to some extent, arbitrary and derive the corresponding control. We discuss the application to the simultaneous control of two non-interacting spin $\frac{1}{2}$ particles with different gyromagnetic ratios in zero field NMR in detail. Our method provides a flexible toolbox for the design of control algorithms to drive the state of finite dimensional quantum systems to any desired final configuration with smooth controls.
\end{abstract}

Keywords: Control Methods for Quantum Mechanical Systems; Smooth Control Functions; Symmetry Reduction; Nuclear Magnetic Resonance.

\section{Introduction}

The combination of control theory techniques with quantum mechanics (see, e.g., 4], 7], [9]) has generated a rich set of control algorithms for quantum mechanical systems modeled by the Schrödinger (operator) equation

$$
\dot{X}=A X+\sum_{j=1}^{m} B_{j} u_{j} X, \quad X(0)=\mathbf{1} .
$$

Here we assume that we have a finite dimensional model, $A$ and $B_{j}$ are matrices in $\mathfrak{s u}(n)$ for each $j=1, \ldots, m, X$ is the unitary propagator, which is equal to the identity $\mathbf{1}$ at time zero, and $u_{j}$ are the controls. These are usually electromagnetic fields, constant in space but possibly time-varying, which are the output of an appropriately engineered pulse-shaping device. Many of the proposed algorithms in the literature involve control functions which are only piecewise continuous and in fact have 'jumps' at certain points of the interval of control. For example, control algorithms based on Lie group decompositions (see, e.g., [13]) involve 'switches' between different Hamiltonians; Algorithms based on optimal control, even if they produce smooth control functions, often require a jump at the beginning of the control interval in order for the control to achieve the prescribed value in norm (assuming a bound in norm of the optimal control as in [5]). Beside the practical problem of generating (almost) instantaneous switches with pulse shapers, such discontinuities introduce undesired high frequency components in the dynamics of the controlled system. For these reasons, it is important to have algorithms which produce smooth control functions whose values at the beginning and the end of the control interval are equal to zero.

This paper describes a method to design control functions without discontinuities in order to drive the state of a class of quantum systems of the form (1) to an arbitrary final configuration. Our main example 
of application will be the simultaneous control of two quantum bits in zero field NMR, a system which was also considered in [10] in the context of optimal control. As compared to that paper, we abandon here the requirement of time optimality (under the requirement of bounded norm for the control) but introduce a novel method which will allow us more flexibility in the control design. The result is a control algorithm that does not present discontinuities, with the control equal to zero at the beginning and at the end of the control interval.

The paper is organized in two main sections each of which divided into several subsections. In Section 2 we describe the class of systems we consider and the general theory underlying our method. We also present two simple examples of quantum systems where the theory applies. In Section 3 we detail the application to the system of two spin $\frac{1}{2}$ particles in zero field NMR above mentioned. This section includes a description of the model as well as the explicit numerical treatment of a control problem: the independent control of the two spin $\frac{1}{2}$ particles to two different types of Hadamard gates.

\section{General Theory}

\subsection{Class of systems considered}

Consider the class of control systems (1) with $A$ and $B_{j}, j=1, \ldots, m$, in $\mathfrak{s u}(n)$ and let $\mathcal{L}$ denote the Lie algebra generated by $\left\{A, B_{1}, \ldots, B_{m}\right\}$. We assume that $\mathcal{L}$ is semi-simple, which implies, since $\mathcal{L} \subseteq \mathfrak{s u}(n)$, that the associated Lie group $e^{\mathcal{L}}$ is compact. The Lie algebra $\mathcal{L}$ is called, in quantum control theory, the dynamical Lie algebra associated to the system (1). Since $e^{\mathcal{L}}$ is compact, the Lie group $e^{\mathcal{L}}$ is the set of states for (1) reachable by changing the control [11]. In particular if $\mathcal{L}=\mathfrak{s u}(n)$, the system is said to be controllable because every special unitary matrix can be obtained with appropriate control. These are known facts in quantum control theory (see, e.g., [7]). We assume that $\mathcal{L}$ has a (vector space) decomposition $\mathcal{L}=\mathcal{K} \oplus \mathcal{P}$, such that $[\mathcal{K}, \mathcal{K}] \subseteq \mathcal{K}$, i.e., $\mathcal{K}$ is a Lie subalgebra of $\mathcal{L}$, which we also assume to be semisimple so that $e^{\mathcal{K}}$ is compact. Moreover $[\mathcal{K}, \mathcal{P}] \subseteq \mathcal{P}$. A special case is when in addition $[\mathcal{P}, \mathcal{P}] \subseteq \mathcal{K}$ in which case the decomposition $\mathcal{L}=\mathcal{K} \oplus \mathcal{P}$ defines a symmetric space of $e^{\mathcal{L}}$ [12]. We assume, in the model (1), that such a decomposition exists so that $A \in \mathcal{K}$ and $\left\{B_{1}, \ldots, B_{m}\right\}$ forms a basis for $\mathcal{P}$.

Under such circumstances, we can reduce ourselves to the case $A=0$ in (1), i.e., to systems of the form

$$
\dot{U}=\sum_{k=1}^{m} \hat{u}_{k} B_{k} U, \quad U(0)=\mathbf{1} .
$$

To see this, assume that for any fixed interval $\left[0, t_{f}\right]$ and any desired final condition $U_{f}$, we are able to find a control $\hat{u}_{k}$ steering the state $U$ in $(2)$ from the identity 1 to $U_{f}$. Let $a_{k j}=a_{k j}(t), k, j=1, \ldots, m$ the coefficients forming an $m \times m$ orthogonal matrix, so that, for any $j=1, \ldots, m$,

$$
e^{-A t} B_{j} e^{A t}=\sum_{k=1}^{m} a_{k j}(t) B_{k} .
$$

Let $X_{f}$ be the desired final condition for (1) and $\hat{u}_{k}$ be the control steering the state $U$ of system (2) from the identity 1 to $e^{-A t_{f}} X_{f}$, in time $t_{f}$. Then the control $u_{j}$ obtained inverting

$$
\hat{u}_{k}(t):=\sum_{j=1}^{m} a_{k j}(t) u_{j}(t),
$$

steers the state $X$ of (1) from the identity to $X_{f}$. This follows from the fact that, if $U=U(t)$ is the solution of (2) with the control $\hat{u}_{k}$, and final condition $e^{-A t_{f}} X_{f}$, then $X=e^{A t} U$ is a solution of (1), with the controls $u_{j}$ given by (4) and therefore the final condition at $t_{f}$ is $X_{f}$. Notice that the transformation 
(4) does not modify the smoothness properties of the control, neither does it modify the fact that the control is zero at the beginning and at the end of the control interval (or at any other point). Therefore in the following we shall deal with driftless systems of the form (2) with the Lie algebraic $\mathcal{L}=\mathcal{K} \oplus \mathcal{P}$, structure above described. In particular $\left\{B_{1}, \ldots, B_{m}\right\}$ forms a basis for $\mathcal{P}$.

\subsection{Symmetry reduction}

The compact Lie group $e^{\mathcal{K}}$ can be seen as a Lie transformation group which acts on $e^{\mathcal{L}}$ via conjugation $X \in e^{\mathcal{L}} \rightarrow K X K^{-1}, K \in e^{\mathcal{K}}$. Moreover this is a group of symmetries for system (2) in the sense that $K B_{j} K^{-1} \in \mathcal{P}$ for each $j$, for every $K \in e^{\mathcal{K}}$. In particular let $K B_{j} K^{-1}:=\sum_{k=1}^{m} a_{k j} B_{k}$ for an orthogonal matrix $\left\{a_{k j}\right\}$ depending on $K \in e^{\mathcal{K}}$ (cf. (3)). If $U=U(t)$ is a trajectory corresponding to a control $\hat{u}_{k}$, $K U K^{-1}$ is the trajectory corresponding to controls $u_{k}:=\sum_{j=1}^{m} a_{k j} \hat{u}_{j}$, as it is easily seen from (2) and

$$
\begin{aligned}
K \dot{U} K^{-1} & =\sum_{j=1}^{m} \hat{u}_{j} K B_{j} K^{-1} K U K^{-1}=\sum_{j=1}^{m} \hat{u}_{j}\left(\sum_{k=1}^{m} a_{k j} B_{k}\right) K U K^{-1}= \\
& =\sum_{k=1}^{m}\left(\sum_{j=1}^{m} a_{k j} \hat{u}_{j}\right) B_{k} K U K^{-1}=\sum_{k=1}^{m} u_{k} B_{k} K U K^{-1} .
\end{aligned}
$$

This suggests to treat the control problem on the quotient space $e^{\mathcal{L}} / e^{\mathcal{K}}$ corresponding to the above action of $e^{\mathcal{K}}$ on $e^{\mathcal{L}}$.

From the theory of Lie transformation groups (see, e.g., [6]) we know that the quotient space $e^{\mathcal{L}} / e^{\mathcal{K}}$ has the structure of a stratified space where each stratum corresponds to an orbit type, i.e., a set of points in $e^{\mathcal{L}}$ which have conjugate isotropy groups. The stratum corresponding to the smallest possible isotropy group, $K_{\text {min }}$, is known to be a connected manifold which is open and dense in $e^{\mathcal{L}} / e^{\mathcal{K}}$. We denote it here by $e_{\text {reg }}^{\mathcal{L}} / e^{\mathcal{K}}$, where reg stands for the regular part. Its preimage in $e^{\mathcal{L}}, e_{\text {reg }}^{\mathcal{L}}$, under the natural projection $\pi: e^{\mathcal{L}} \rightarrow e^{\mathcal{L}} / e^{\mathcal{K}}$ is open and dense in $e^{\mathcal{L}}$ as well. This is called the regular part of $e^{\mathcal{L}} / e^{\mathcal{K}},\left(\right.$ resp. $\left.e^{\mathcal{L}}\right)$. The complementary set in $e^{\mathcal{L}} / e^{\mathcal{K}},\left(\right.$ resp. $\left.e^{\mathcal{L}}\right)$ is called the singular part. The dimension of $e_{\text {reg }}^{\mathcal{L}} / e^{\mathcal{K}}$ as a manifold is

$$
\operatorname{dim}\left(e_{\text {reg }}^{\mathcal{L}} / e^{\mathcal{K}}\right)=\operatorname{dim}\left(e^{\mathcal{L}}\right)-\operatorname{dim}\left(e^{\mathcal{K}}\right)+\operatorname{dim} K_{\text {min }}=\operatorname{dim}(\mathcal{L})-\operatorname{dim}(\mathcal{K})+\left(\operatorname{dim} K_{\text {min }}\right),
$$

where $\operatorname{dim}\left(K_{\min }\right)$ is the dimension of the minimal isotropy group as a Lie group ${ }^{1}$ In particular, if $K_{\min }$ is a discrete Lie group, i.e., it has dimension zero, the right hand side of (5) is the dimension of the subspace $\mathcal{P}$. This is verified for instance in $K-P$ problems (cf., e.g., [8]) when $e^{\mathcal{L}}=S U(n)$. We shall assume this to be the case in the following.

According to a result in [8], under the assumption that the minimal isotropy group $K_{\min }$ is discrete, the restriction of $\pi_{*}$ to $R_{x *} \mathcal{P}$ is an isomorphism onto $T_{\pi(x)}\left(e_{\text {reg }}^{\mathcal{L}} / e^{\mathcal{K}}\right)$ for each point $x$ in the regular part, $e_{\text {reg }}^{\mathcal{L}}$. Here, as it is often done, we have identified the Lie algebra $\mathcal{L}$ with the tangent space of $e^{\mathcal{L}}$ at the identity $\mathbf{1}$, and therefore $\mathcal{P}$ is identified with a subspace of the tangent space at $\mathbf{1}$. The map $R_{x}$ denotes the right translation by $x$ so that $R_{x *} \mathcal{P}$ is a subspace (with the same dimension) of the tangent space at $x, T_{x} e^{\mathcal{L}} 2$ In Appendix B, we show that in given coordinates the determinant of the restriction of $\pi_{*}$ to $R_{x *} \mathcal{P}$ is invariant under the action of $e^{\mathcal{K}}$. The above isomorphism result says that in the regular part $\operatorname{det} \pi_{*} \neq 0$. In this situation, for every regular point $U \in e^{\mathcal{L}}$, for every tangent vector $V \in T_{\pi(U)}\left(e_{\text {reg }}^{\mathcal{L}} / e^{\mathcal{K}}\right)$, we can find a tangent vector $\pi_{*}^{-1} V \in R_{U *} \mathcal{P}$. Such a tangent vector is horizontal for system (2) which

\footnotetext{
${ }^{1}$ More discussion on these basic facts in the theory of Lie transformation groups can be found in [1] and references therein.

${ }^{2}$ Recall that for a map $f: M \rightarrow N$ for two manifolds $M$ and $N, f_{*}$ denotes the differential (also called push-forward) $f_{*}: T_{x} M \rightarrow T_{f(x)} N$ between two tangent spaces. When we want to emphasize the point $x$ we write $\left.f_{*}\right|_{x}$.
} 
means that it can be written as a linear combination of the available vector fields $\left\{B_{k} U\right\}$ in (2). If $\Gamma=\Gamma(t)$ is a curve entirely contained in $e_{\text {reg }}^{\mathcal{L}} / e^{\mathcal{K}}$ and $U=U(t)$ a curve in $e_{\text {reg }}^{\mathcal{L}}$ such that $\pi(U(t))=\Gamma(t)$ for every $t$, i.e., $U$ is a 'lift' of $\Gamma$, then $\left.\pi_{*}\right|_{U} ^{-1} \dot{\Gamma}$ is a horizontal tangent vector at $U(t)$ for every $t$. If $\Gamma$ joins two points $\Gamma_{0}$ and $\Gamma_{1}$ in $e_{\text {reg }}^{\mathcal{L}} / e^{\mathcal{K}}$, in the interval $\left[t_{0}, t_{1}\right]$, and $U_{0}$ is such that $\pi\left(U_{0}\right)=\Gamma_{0}$, then the solution of the differential system

$$
\dot{U}=\left.\pi_{*}\right|_{U} ^{-1} \dot{\Gamma}, \quad U\left(t_{0}\right)=U_{0},
$$

is such that $\pi\left(U\left(t_{1}\right)\right)=\Gamma_{1}$. Therefore, once we prescribe an arbitrary trajectory $\Gamma$ to move in the quotient space between two given orbits $\Gamma_{0}$ and $\Gamma_{1}$ in the regular part, the control specified by

$$
\left.\pi_{*}\right|_{U} ^{-1} \dot{\Gamma}=\sum_{j=1}^{m} u_{j} B_{j} U
$$

will allow us to move between two states $U_{0}$ and $U_{1}$ such that $\pi\left(U_{0}\right)=\Gamma_{0}$ and $\pi\left(U_{1}\right)=\Gamma_{1}$.

\subsection{Methodology for Control}

The above treatment suggests a general methodology to design control laws for systems of the form (2) described in subsection 2.1. In fact, given the freedom in the choice of the trajectory $\Gamma=\Gamma(t)$ above mentioned, we can design such controls satisfying various requirements and in particular without discontinuity. Such a methodology can be summarized as follows.

First of all we need to obtain a geometric description of the orbit space $e^{\mathcal{L}} / e^{\mathcal{K}}$, and in particular of its regular part $e_{\text {reg }}^{\mathcal{L}} / e^{\mathcal{K}}$, and verify that the minimal isotropy group, which is the isotropy group of the elements in $e_{\text {reg }}^{\mathcal{L}}$, is discrete so that the right hand side of (5) is equal to $\operatorname{dim} \mathcal{P}$. This is a weak assumption, easily verified in the examples that will follow and that can be proven true in several cases [8], 14. Then one chooses coordinates for the manifold $e_{\text {reg }}^{\mathcal{L}} / e^{\mathcal{K}}$. These are expressed in terms of the original coordinates in $e^{\mathcal{L}}$ or, more commonly, in terms of the entries of the matrices in $e^{\mathcal{L}}$. Such coordinates are a complete set of independent invariants with respect to the (conjugacy) action of the group $e^{\mathcal{K}}$. The word 'complete' here means that the knowledge of their values uniquely determines the orbit, i.e., a point in $e_{\text {reg }}^{\mathcal{L}} / e^{\mathcal{K}}$. There are $m=\operatorname{dim}(\mathcal{P})$ of them, as this is the dimension of $e_{\text {reg }}^{\mathcal{L}} / e^{\mathcal{K}}$ (cf. (5)). Once we have coordinates $\left\{x^{1}, \ldots, x^{m}\right\}$, the tangent vectors $\left\{\frac{\partial}{\partial x^{1}}, \ldots, \frac{\partial}{\partial x^{m}}\right\}$ at every regular point in the quotient space determine a basis of the tangent space of $e_{\text {reg }}^{\mathcal{L}} / e^{\mathcal{K}}$. For any trajectory $\Gamma$ in $e_{\text {reg }}^{\mathcal{L}} / e^{\mathcal{K}}$, we can write the tangent vector $\dot{\Gamma}(t)$ as $\dot{\Gamma}(t)=\sum_{j=1}^{m} \dot{x}^{j} \frac{\partial}{\partial x^{j}}$, for some functions $\dot{x}^{j}$. With this choice of coordinates, one then needs to calculate, for every regular point $U$, the matrix for $\left.\pi_{*}\right|_{U}$ as restricted to $R_{U *} \mathcal{P}$ and its inverse $\left.\pi_{*}\right|_{U} ^{-1}$. This allows us to implement formula (7) to obtain the control from a given prescribed trajectory in the orbit space.

We remark that there is an issue concerning the fact that our initial condition which is the identity 1 in 22 (and possibly the final desired condition) is not in the regular part of $e^{\mathcal{L}}$. In fact the whole Lie group $e^{\mathcal{K}}$ is the isotropy group of the identity. If we take for $\Gamma$ a trajectory which starts from the class corresponding to the identity, the matrix corresponding to $\pi_{*}$ may become singular as $t \rightarrow 0$ and therefore it will be impossible to derive the control directly from formula (7). This problem can be overcome by applying a preliminary control which takes the state of system (2) out of the singular part and into the regular part of $e^{\mathcal{L}}$. To avoid discontinuities, such a control is chosen to be zero at the beginning and at the end of the control interval. It takes the system to a point $U_{0}$ with $\pi\left(U_{0}\right)=\Gamma_{0} \in e_{\text {reg }}^{\mathcal{L}} / e^{\mathcal{K}}$. Then we choose the trajectory in the quotient space $\Gamma$ in the regular part of the quotient space which joins $\Gamma_{0}$ and $\Gamma_{1}$ where $\Gamma_{1}$ is the orbit of the desired final condition. The control obtained through (7) will steer system (2) to a state $\hat{U}_{f}$ in the same orbit as the desired final condition. Therefore if $U_{f}$ is the desired final condition we will have $\pi\left(\hat{U}_{f}\right)=\pi\left(U_{f}\right)=\Gamma_{1}$. Notice that we also want $\dot{\Gamma} \rightarrow 0$ at both the initial and 
final point so that the control is zero and can be concatenated continuously with the preliminary control above described.

It is possible that the desired final condition $U_{f}$ is also in the singular part of $e^{\mathcal{L}}$. This problem can be tackled in two ways. We can recall that the regular part is open and dense and therefore we can always drive to a state in the regular part arbitrarily close to the desired $U_{f}$. This means that our algorithm will only give an approximate control, but which will steer the system arbitrarily close to $U_{f}$. Alternatively we can select a regular element $S \in e^{\mathcal{L}}$ and such that $U_{f} S^{-1}$ is also regular. ${ }^{3}$ Then we find two controls: $u_{1}$ driving $U$ in 2 from the identity to $S$ in $(2)$ and $u_{2}$ driving $U$ in (2) from the identity to $U_{f} S^{-1}$ in (2). In particular, because of the right invariance of system (2), $u_{2}$ also drives $S$ to $U_{f}$. Therefore, the concatenation of $u_{1}$ (first) and $u_{2}$ (second) will drive to the desired final configuration. Therefore in the following, for simplicity, we shall assume that the final desired state is in the regular part.

The (concatenated) control $\hat{u}_{j}$ obtained from the tangent vector $\dot{\Gamma}$ at every time $t$ for a trajectory on the quotient space $\Gamma$ (cf. $(7)$ ) will drive the state $U$ of $(2)$ from the identity $\mathbf{1}$ only to a state $\hat{U}_{f}$ which is in the same orbit as the desired final state $U_{f}$. There exists $K \in e^{\mathcal{K}}$ such that $K \hat{U}_{f} K^{-1}=U_{f}$. Once such a $K$ is found it will determine through $\sum_{j=1}^{m} K B_{j} \hat{u}_{j} K^{-1}=\sum_{k=1}^{m} B_{k} u_{k}$ the actual control $\left\{u_{k}\right\}$ to apply. We remark that this tranformation does not modify the smoothness properties of the control, nor the fact that it is zero at some point (in particular at the beginning and at the end of the control interval).

\subsection{Examples}

\subsubsection{Control of a single spin $\frac{1}{2}$ particle}

Consider the Schrödinger operator equation (2) in the form

$$
\dot{U}=\left(\begin{array}{cc}
0 & \alpha(t) \\
-\alpha^{*}(t) & 0
\end{array}\right) U, \quad U(0)=\mathbf{1}_{2}
$$

with $U$ in $S U(2)$. The complex-valued function $\alpha$ is a complex control field representing the $x$ and $y$ components of an electro-magnetic field. The dynamical Lie algebra $\mathcal{L}$ is $\mathfrak{s u}(2)$ and it has a decomposition $\mathfrak{s u}(2)=\mathcal{K} \oplus \mathcal{P}$ with $\mathcal{K}$ diagonal and $\mathcal{P}$ anti-diagonal matrices. The one-dimensional Lie group of diagonal matrices in $S U(2)=e^{\mathcal{L}}$ is a symmetry group $e^{\mathcal{K}}$ for the above system and the structure of the quotient space $S U(2) / e^{\mathcal{K}}$ is that of a closed unit disc [2]. The entry $u_{1,1}$ of $U \in S U(2)$, which is a complex number with absolute value $\leq 1$, determines the orbit of the matrix $U$. The regular part of $S U(2)$ corresponds to matrices with $\left|u_{1,1}\right|<1$, i.e., the interior of the unit disc $\stackrel{\circ}{D}$, in the complex plane. The singular part is the boundary of the unit disc. Denote by $z$ the (complex) coordinate in the interior of the complex unit disc. This corresponds to two real coordinates invariant under the action of $e^{\mathcal{K}}$ (conjugation by diagonal matrices). Let $\Gamma=\Gamma(t)$ be a desired trajectory inside the unit disc, which we denote by $z_{d}$ in the chosen coordinates. The tangent vector $\dot{\Gamma}$ in $(6)$ is given in complex coordinates by $\dot{z}_{d} \frac{\partial}{\partial z} \bigsqcup^{4}$ In the coordinates on $S U(2)$ used in equation $\left[22\right.$ the corresponding tangent vector for $\dot{U}$ is given by (cf. $\left[\begin{array}{l}8 \\ \text { ) }\end{array}\right)\left(\begin{array}{cc}0 & \alpha \\ -\alpha & 0\end{array}\right) U$

\footnotetext{
${ }^{3}$ Such an element $S \in e_{\text {reg }}^{\mathcal{L}}$ always exists for any $U \in e^{\mathcal{L}}$, by the following argument: Assume that it does not exist. Then for every regular $S, U S$ is singular. Therefore, by indicating by $L_{U}$ the left translation by $U$ we have, $L_{U}\left(e_{\text {reg }}^{\mathcal{L}}\right) \subseteq e_{\text {sing }}^{\mathcal{L}}$. Then by applying the unique bi-invariant Haar measure $\mu$ on $e^{\mathcal{L}}$ with $\mu\left(e^{\mathcal{L}}\right)=1$ implies $\mu\left(e_{\text {reg }}^{\mathcal{L}}\right)=\mu\left(L_{U}\left(e_{\text {reg }}^{\mathcal{L}}\right)\right) \leq \mu\left(e_{\text {sing }}^{\mathcal{L}}\right)$. On the other hand, $\mu\left(e_{\text {sing }}^{\mathcal{L}}\right)=0$ since $\mu$ must also correspond to the Riemannian volume of the bi-invariant Killing metric (normalized if necessary) and each stratum in $e_{\text {sing }}^{\mathcal{L}}$ has dimension strictly less than dimension of $e^{\mathcal{L}}$ and thus has volume 0 and therefore invariant measure 0 . But $\mu\left(e_{\text {reg }}^{\mathcal{L}}\right)=\mu\left(e^{\mathcal{L}}\right)-\mu\left(e_{\text {sing }}^{\mathcal{L}}\right)=\mu\left(e^{\mathcal{L}}\right)=1$. This is a contradiction.

${ }^{4}$ This means $\dot{x}_{d} \frac{\partial}{\partial x_{d}}+\dot{y}_{d} \frac{\partial}{\partial y_{d}}$ where $x_{d}$ and $y_{d}$ are the real and imaginary parts of $z_{d}$.
} 
and the value of the control $\alpha$ is obtained by solving (6) which gives

$$
\dot{z}_{d}=\left.\frac{d}{d t}\right|_{t=0} z\left(e^{\left(\begin{array}{cc}
0 & \alpha \\
-\alpha^{*} & 0
\end{array}\right)_{t}} U\right)
$$

where $z(P)$ denotes the the $(1,1)$ entry of the matrix $P$. Equation $\sqrt{9}$ gives $\alpha=\frac{\dot{z}_{d}}{u_{21}}$, which, as expected from the above recalled isomorphism theorem of [8], gives a one-to-one correspondence between $\alpha$ and $\dot{z}_{d}$ as long as $U$ is in the regular part of $S U(2)$, i.e., it is not diagonal, i.e., $u_{2,1} \neq 0$.

\subsection{Control of a three level system in the $\Lambda$ configuration}

Consider a three level quantum system where the controls couple level $|1\rangle$ to level $|2\rangle$ and level $|1\rangle$ to level $|3\rangle$ but not level $|2\rangle$ and $|3\rangle$ directly. Assuming that $|1\rangle$ is the highest energy level, the energy level diagram takes the so-called $\Lambda$ configuration (see, e.g., [15]). The Schrödinger operator equation (2) is such that

$$
\sum_{j=1}^{m} u_{j} B_{j}=\sum_{j=1}^{4} u_{j} B_{j}=\left(\begin{array}{ccc}
0 & \alpha & \beta \\
-\alpha^{*} & 0 & 0 \\
-\beta^{*} & 0 & 0
\end{array}\right)
$$

with the complex control functions $\alpha$ and $\beta$. Such a system admits a group of symmetries given by $e^{\mathcal{K}}=S(U(1) \times U(2))$, i.e., block diagonal matrices in $S U(3)$ with one block of dimension $1 \times 1$ and one block of dimension $2 \times 2$, and determinant equal to 1 . The Lie subalgebra $\mathcal{K}$ consists of matrices in $\mathfrak{s u}(3)$ with a block diagonal structure with one block of dimension $1 \times 1$ and one block of dimension $2 \times 2$. The complementary subspace $\mathcal{P}$ is spanned by antidiagonal matrices in $\mathfrak{s u}(2)$ with the same partition of rows and columns. Such a system was studied in [3] in the context of optimal control and a description of the orbit space $S U(3) / e^{\mathcal{K}}$ was given. It was shown that the regular part $S U(3)_{r e g} / e^{\mathcal{K}}$ is homeomorphic to the product of two open unit discs $\stackrel{\circ}{D}_{1} \times \stackrel{\circ}{D}_{2}$ in the complex plane. Up to a similarity transformation in $e^{\mathcal{K}}=S(U(1) \times U(2))$, a matrix $U$ in $S U(3)$ can be written as

$$
U=\left(\begin{array}{ccc}
z_{1} & \sqrt{1-\left|z_{1}\right|^{2}} & 0 \\
-\sqrt{1-\left|z_{1}\right|^{2}} & z_{1}^{*} & 0 \\
0 & 0 & 1
\end{array}\right)\left(\begin{array}{ccc}
1 & 0 & 0 \\
0 & z_{2} & w \\
0 & -w^{*} & z_{2}^{*}
\end{array}\right)
$$

for complex numbers $z_{1}, z_{2}$ and $w$, where $\left|z_{1}\right| \leq 1$ and $\left|z_{2}\right| \leq 1$. Strict inequalities hold if and only if $U$ is in the regular part in which case $z_{1}$ and $z_{2}$ can be taken as the coordinates in $S U(3)_{\text {reg }} / e^{\mathcal{K}}$. An alternative set of (complex) coordinates is given by $\left(z_{1}, T\right)$ where $T$ is the trace of the (lower) $2 \times 2$ block of the element $U \in S U(3)$ which is invariant (along with $z_{1}$ ) under the conjugation action of elements in $e^{\mathcal{K}}=S(U(1) \times U(2))$. The coordinates $\left(z_{1}, T\right)$ are related to the coordinates $\left(z_{1}, z_{2}\right)$ by (from $\left.[11)\right)$ $T=z_{1}^{*} z_{2}+z_{2}^{*}$ which is inverted as $z_{2}=\frac{T^{*}-z_{1} T}{1-\left|z_{1}\right|^{2}}$. A desired trajectory $\Gamma$ in $S U(3)_{r e g} / S(U(1) \times U(2))$ is written in these coordinates as $\left(z_{1 d}, T_{d}\right):=\left(z_{1 d}(t), T_{d}(t)\right)$. The associated tangent vector $\dot{\Gamma}$ in $(6)$ is $\dot{z}_{1 d}(t) \frac{\partial}{\partial z_{1 d}}+\dot{T}_{d} \frac{\partial}{\partial T}$. By applying $\left.\pi_{*}\right|_{U} \dot{U}$ in $\sqrt{6}$ to $z_{1}$ and $T$ with the restriction that $\dot{U}$ is of the form $\left(\begin{array}{ccc}0 & \alpha & \beta \\ -\alpha^{*} & 0 & 0 \\ -\beta^{*} & 0 & 0\end{array}\right) U$, we obtain two equations for $\alpha$ and $\beta$,

$$
\alpha u_{2,1}+\beta u_{3,1}=\dot{z}_{1 d}, \quad-\alpha u_{1,2}^{*}-\beta u_{1,3}^{*}=\dot{T}_{d}^{*}
$$


These are solved, using $\hat{D}:=u_{1,3}^{*} u_{2,1}-u_{3,1} u_{1,2}^{*}$ by

$$
\alpha=\frac{u_{1,3}^{*} \dot{z}_{1, d}+u_{3,1} \dot{T}_{d}^{*}}{\hat{D}}, \quad \beta=-\frac{u_{1,2}^{*} \dot{z}_{1, d}+u_{2,1} \dot{T}_{d}^{*}}{\hat{D}} .
$$

The quantity $\hat{D}$ is different from zero if and only if the matrix $U$ is in the regular part of $S U(3)$. This can be shown in two steps: First one shows that $\hat{D}$ is invariant under the action of $S(U(1) \times U(2))$ by writing a matrix in $S(U(1) \times U(2))$ with an Euler-type decomposition as $F_{1} R F_{2}$ with $F_{1}$ and $F_{2}$ diagonal and $R$ of the form $R=\left(\begin{array}{ccc}1 & 0 & 0 \\ 0 & \cos (\theta) & \sin (\theta) \\ 0 & -\sin (\theta) & \cos (\theta)\end{array}\right)$, and verifying that conjugation by each factor in $F_{1} R F_{2}$ leaves $\hat{D}$ unchanged. The second step is to verify that $\hat{D}$ for the matrix $X$ in the form 11 is different from zero if and only if $\left|z_{1}\right| \neq 1$ and $\left|z_{2}\right| \neq 1$. This gives a quick test to check whether a matrix is in the regular part, i.e., if its isotropy group is the smallest possible one, which, in this case, is the group of scalar matrices in $S U(3)$. This fact also follows from the result in Appendix B which shows in general that $\operatorname{det}\left(\pi_{*}\right)$, with $\pi_{*}: R_{p *} \mathcal{P} \rightarrow T_{\pi(p)} e_{\text {reg }}^{\mathcal{L}} / e^{\mathcal{K}}$ is invariant under the action of $e^{\mathcal{K}}$.

As always, we have the problem that the initial point $\mathbf{1}$ is in the singular part of the orbit space decomposition and therefore $\hat{D}$ in $\sqrt{12}$ is zero at time 0 . As suggested in the previous section, we can however apply a preliminary control to steer away from the singular part.

\section{Simultaneous control of two independent spin $\frac{1}{2}$ particles}

\subsection{The model}

The dynamics of two spin $\frac{1}{2}$ particles with different gyromagnetic ratios in zero field NMR can be described by the Schrödinger equation (2) (after appropriate normalization) where

$$
\sum_{k=1}^{m} \hat{u}_{k} B_{k}:=\sum_{x, y, z} u_{x, y, z}(t)\left(i \sigma_{x, y, z} \otimes \mathbf{1}+\gamma i \mathbf{1} \otimes \sigma_{x, y, z}\right) .
$$

Here $u_{x, y, z}$ are the controls representing the $x, y, z$ components of the electromagnetic field, and $\sigma_{x, y, z}$ are the Pauli matrices defined as

$$
\sigma_{x}:=\left(\begin{array}{cc}
0 & 1 \\
1 & 0
\end{array}\right), \quad \sigma_{y}:=\left(\begin{array}{cc}
0 & -i \\
i & 0
\end{array}\right), \quad \sigma_{z}:=\left(\begin{array}{cc}
1 & 0 \\
0 & -1
\end{array}\right) .
$$

The parameter $\gamma$ is the ratio of the two gyromagnetic ratios and we shall assume that $|\gamma| \neq 1$. Under this assumption, the dynamical Lie algebra $\mathcal{L}$ for system (2), (13) is the 6-dimensional Lie algebra spanned by $\left\{\sigma_{1} \otimes \mathbf{1}+\mathbf{1} \otimes \sigma_{2} \mid \sigma_{1}, \sigma_{2} \in \mathfrak{s u}(2)\right\} .^{5}$ The corresponding Lie group $e^{\mathcal{L}}$, which is the set of reachable states for system (2), (13), is $\left\{X_{1} \otimes X_{2} \mid X_{1}, X_{2} \in S U(2)\right\}$, i.e. the tensor product $S U(2) \otimes S U(2)$. It is convenient to slightly relax the description of the state space and look at system (2), (13) as a system on the Lie group $S U(2) \times S U(2)$, i.e., the Cartesian direct product of $S U(2)$ with itself, and the dynamical equations (2), (13) replaced by

$$
\dot{U}=\sigma(t) U, \quad U(0)=\mathbf{1}, \quad \dot{V}=\gamma \sigma(t) V, \quad V(0)=\mathbf{1},
$$

with $\sigma(t):=\sum_{x, y, z} i u_{x, y, z}(t) \sigma_{x, y, z}$. The controls that drive system (15) to $\left( \pm U_{f}, \pm V_{f}\right)$ drive system (2), (13) to the state $U_{f} \otimes V_{f}$. Therefore we shall focus on the steering problem for system (15) which consists

\footnotetext{
${ }^{5}$ This Lie algebra is isomorphic to $\mathfrak{s o}(4)$.
} 
of steering one spin to $U_{f}$ and the other to $V_{f}$, simultaneously. Since $|\gamma| \neq 1$, the dynamical Lie algebra associated with (15) is spanned by the pairs $\left(\sigma_{1}, \sigma_{2}\right)$ with $\sigma_{1}$ and $\sigma_{2}$ in $\mathfrak{s u}(2)$. Such a Lie algebra can be written as $\mathcal{K} \oplus \mathcal{P}$ with $\mathcal{K}$ spanned by elements of the form $(\sigma, \sigma)$ with $\sigma \in \mathfrak{s u}(2)$ and $\mathcal{P}$ spanned by elements of the form $(\sigma, \gamma \sigma)$ with $\sigma \in \mathfrak{s u}(2)$. At every $p \in G=S U(2) \times S U(2)$, the vector fields in (15) belong to $R_{p *} \mathcal{P}$.

\subsection{Symmetries and the the structure of the quotient space}

The Lie group $S U(2)$ acts on $G:=S U(2) \times S U(2)$ by simultaneous conjugation, that is, for $K \in S U(2)$, $\left(U_{f}, V_{f}\right) \rightarrow\left(K U_{f} K^{-1}, K V_{f} K^{-1}\right)$ and this is a group of symmetries for system 15$)$ in that if $\sigma=\sigma(t)$ is the control steering to $\left(U_{f}, V_{f}\right)$, then $K \sigma K^{-1}$ is the control steering to $\left(K U_{f} K^{-1}, K V_{f} K^{-1}\right)$. The quotient space of $S U(2) \times S U(2)$ under this action, $(S U(2) \times S U(2)) / S U(2)$ was described in [10] as follows.

Consider a pair $\left(U_{f}, V_{f}\right)$ and let $\phi \in[0, \pi]$ be a real number so that the two eigenvalues of $U_{f}$ are $e^{i \phi}$ and $e^{-i \phi}$. If $0<\phi<\pi$ then $U_{f} \neq \pm \mathbf{1}$ and there exists a unitary matrix $S$ such that $S U_{f} S^{\dagger}:=D_{f}$ is diagonal. Therefore the matrix $\left(U_{f}, V_{f}\right)$ is in the same orbit as $\left(D_{f}, S V_{f} S^{\dagger}\right)$. The parameter $\phi$ determines the orbit, along with the $(1,1)$-entry of $S V_{f} S^{\dagger}$, which does not depend on the choice of $S !^{6}$ Such a $(1,1)$ entry has absolute value $\leq 1$ and therefore it is an element of the unit disk in the complex plane. The orbits corresponding to the values of $0<\phi<\pi$ (for the eigenvalue of the first matrix) are in one-to-one correspondence with the points of a solid cylinder with height equal to $\phi$. When $\phi=0$ (or $\phi=\pi$ ), the matrix $U_{f}$ is \pm identity and therefore the equivalence class is determined by the eigenvalue of the matrices $V_{f}$, which are $e^{ \pm i \psi}$ for $\psi \in[0, \pi]$. In the geometric description, the solid cylinder degenerates at the two ends to become a segment $[0, \pi]$. The regular part of the orbit space $G_{r e g}$ is represented by points in the interior of the solid cylinder. Such points correspond to pairs

$$
\left(\left(\begin{array}{cc}
e^{i \phi} & 0 \\
0 & e^{-i \phi}
\end{array}\right),\left(\begin{array}{cc}
z & w \\
-w^{*} & z^{*}
\end{array}\right)\right)
$$

with $\phi \in(0, \pi)$ and $|z|<1$. For these pairs, the isotropy group is the discrete group $\{ \pm \mathbf{1}\}$. In general points that are in the singular part correspond to pairs of matrices $\left(U_{f}, V_{f}\right)$ which can be simultaneously diagonalized. Therefore the condition that they commute

$$
U_{f} V_{f}=V_{f} U_{f},
$$

is necessary and sufficient for a pair $\left(U_{f}, V_{f}\right)$ to belong to the singular part.

Assume that $p$ is a regular point in $G_{r e g}$ for this problem and $\pi$ is the natural projection $\pi: G_{r e g} \rightarrow$ $G_{r e g} / S U(2)$. Then from the theory in the previous section, the differential $\left.\pi_{*}\right|_{p}$ is an isomorphism from $R_{p *} \mathcal{P}$ to $T_{\pi(p)} G_{r e g} / S U(2)$. Let us choose a basis for $\mathcal{P}$ given by $\left(i \sigma_{x, y, z}, \gamma i \sigma_{x, y, z}\right)$. To choose the three coordinates in $G_{r e g} / S U(2)$, we consider a general element $p$ in $S U(2) \times S U(2)$ written as

$$
p:=\left(U_{f}, V_{f}\right):=\left(\left(\begin{array}{cc}
x & y \\
-y^{*} & x^{*}
\end{array}\right),\left(\begin{array}{cc}
z & w \\
-w^{*} & z^{*}
\end{array}\right)\right) .
$$

For a complex number $q$ we shall denoted by $q_{R}:=\operatorname{Re}(q)$ and $q_{I}:=\operatorname{Im}(q)$. Notice that in (17) we have

$$
x_{R}^{2}+x_{I}^{2}+y_{R}^{2}+y_{I}^{2}=z_{R}^{2}+z_{I}^{2}+w_{R}^{2}+w_{I}^{2}=1 .
$$

Coordinates in $G_{r e g} / S U(2)$ must be independent invariant functions of $\left(U_{f}, V_{f}\right)$ in (17). We choose

$$
x^{1}:=x_{R}, \quad x^{2}:=z_{R}, \quad x^{3}:=x_{I} z_{I}+w_{R} y_{R}+w_{I} y_{I} .
$$

\footnotetext{
${ }^{6}$ All the possible diagonalizing matrices differ by a diagonal factor that does not affect the $(1,1)$ entry of $S V_{f} S^{\dagger}$.
} 
It is a direct verification to check that at any point $p \in S U(2) \times S U(2), x^{1}, x^{2}$ and $x^{3}$ are unchanged by the (double conjugation) action of $S U(2)$, i.e., they are invariant. We remark also that we can consider two unit vectors $\vec{V}:=\left(x_{R}, x_{I}, y_{R}, y_{I}\right)$, and $\vec{W}:=\left(z_{R}, z_{I}, w_{R}, w_{I}\right)$, and, if we do that, $x^{3}=\vec{V} \cdot \vec{W}-x_{R} z_{R}$.

\subsection{Choice of invariants}

We pause a moment to detail how the invariant coordinates in 18 were chosen. We do this because the method can be used for other examples. We consider the vectors $\vec{V}:=\left[x_{R}, x_{I}, y_{R}, y_{I}\right]^{T}$ and $\vec{W}:=$ $\left[z_{R}, z_{I}, w_{R}, w_{I}\right]^{T}$ and the adjoint action of $S U(2)$ on $S U(2) \times S U(2)$ which gives a linear action on $\vec{Q}:=\left[\vec{V}^{T}, \vec{W}^{T}\right]^{T}$. We are looking for functions $f=f(\vec{V}, \vec{W})$ invariant under this action. Given that every element of $S U(2)$ can be written according to Euler's decomposition as $e^{i \sigma_{z} \alpha} e^{i \sigma_{y} \theta} e^{i \sigma_{z} \beta}$, for real parameters $\alpha, \beta$ and $\theta$, it is enough that $f$ is invariant with respect to transformations of the form $e^{i \sigma_{z} \beta}$ and $e^{i \sigma_{y} \theta}$, for general real $\beta$ and $\theta$, in order for $f$ to be invariant with respect to all of $S U(2)$. If $X_{z}:=X_{z}(\beta):=e^{i \sigma_{z} \beta}$ then $A d_{X_{z}}$ acting on $\left[\vec{V}^{T}, \vec{W}^{T}\right]^{T}$ is

$$
A d_{X_{z}(\beta)}:=\left(\begin{array}{cccc}
1 & 0 & 0 & 0 \\
0 & 1 & 0 & 0 \\
0 & 0 & \cos (\beta) & -\sin (\beta) \\
0 & 0 & \sin (\beta) & \cos (\beta)
\end{array}\right) .
$$

If $X_{y}:=X_{y}(\theta):=e^{i \sigma_{y} \theta}$ then $A d_{X_{y}}$ acting on $\left[\vec{V}^{T}, \vec{W}^{T}\right]^{T}$ is

$$
A d_{X_{y}(\theta)}:=\left(\begin{array}{cccc}
1 & 0 & 0 & 0 \\
0 & \cos (\theta) & 0 & \cos (\theta) \\
0 & 0 & 1 & 0 \\
0 & -\sin (\theta) & 0 & \cos (\theta)
\end{array}\right) .
$$

We first look for linear invariants, i.e., invariant functions $f$ of the form $f(\vec{V}, \vec{W}):=\vec{a}^{T} \vec{V}+\vec{b}^{T} \vec{W}$. From the condition

$$
\vec{a}^{T} \vec{V}+\vec{b}^{T} \vec{W}=\vec{a}^{T} A d_{X} \vec{V}+\vec{b}^{T} A d_{X} \vec{W},
$$

where $X$ may be equal to $X_{z}(\beta)$ or $X_{y}(\theta)$, with arbitrary $\beta$ and $\theta$, we find that the last three components of $\vec{a}$ and $\vec{b}$ must be zero. Therefore all linear invariants $f$ must be of the form $f=a_{1} x_{R}+b_{1} z_{R}$, from which we get the invariant $x_{R}$ and $z_{R}$ in 18 .

We then try to find quadratic invariants and therefore an $8 \times 8$ symmetric matrix $P$ so that $f(\vec{V}, \vec{W})=$ $\left[\vec{V}^{T}, \vec{W}^{T}\right] P\left[\vec{V}^{T}, \vec{W}^{T}\right]^{T}$ and

$$
\left[\vec{V}^{T}, \vec{W}^{T}\right] P\left[\vec{V}^{T}, \vec{W}^{T}\right]^{T}=\left[\vec{V}^{T}, \vec{W}^{T}\right]\left(\begin{array}{cc}
A d_{X}^{T} & 0 \\
0 & A d_{X}^{T}
\end{array}\right) P\left(\begin{array}{cc}
A d_{X} & 0 \\
0 & A d_{X}
\end{array}\right)\left[\vec{V}^{T}, \vec{W}^{T}\right]^{T},
$$

for $X=X_{z}(\beta)$ and $X=X_{y}(\theta)$ as defined in $(19)$ and $(20)$ for every $\beta$ and $\theta$ (and for every $\vec{V}$ and $\vec{W}$ ). This leads to the condition

$$
\left(\begin{array}{cc}
A d_{X} & 0 \\
0 & A d_{X}
\end{array}\right) P=P\left(\begin{array}{cc}
A d_{X} & 0 \\
0 & A d_{X}
\end{array}\right) .
$$


From this, we find that the matrix $P$ must be of the form

$$
P=\left(\begin{array}{llllllll}
e & 0 & 0 & 0 & d & 0 & 0 & 0 \\
0 & c & 0 & 0 & 0 & g & 0 & 0 \\
0 & 0 & c & 0 & 0 & 0 & g & 0 \\
0 & 0 & 0 & c & 0 & 0 & 0 & g \\
d & 0 & 0 & 0 & r & 0 & 0 & 0 \\
0 & g & 0 & 0 & 0 & h & 0 & 0 \\
0 & 0 & g & 0 & 0 & 0 & h & 0 \\
0 & 0 & 0 & g & 0 & 0 & 0 & h
\end{array}\right)
$$

It follows that all quadratic invariants must be of the form

$$
f=e x_{R}^{2}+2 d x_{R} z_{R}+r z_{R}^{2}+c\left(x_{I}^{2}+y_{R}^{2}+y_{I}^{2}\right)+h\left(z_{I}^{2}+w_{R}^{2}+w_{I}^{2}\right)+2 g\left(x_{I} z_{I}+w_{R} y_{R}+w_{I} y_{I}\right) .
$$

Because of $x_{R}^{2}+x_{I}^{2}+y_{R}^{2}+y_{I}^{2}=z_{R}^{2}+z_{I}^{2}+w_{R}^{2}+w_{I}^{2}=1$, all terms can be written in terms of the (linear) invariant $x_{R}$ and $z_{R}$ except the last one which we choose as the third coordinate in (18).

\subsection{Algorithm for control}

At the point $\pi(p) \in G_{r e g} / S U(2)$, the tangent vectors $\frac{\partial}{\partial x^{j}}, j=1,2,3$ span $T_{\pi(p)} G_{r e g} / S U(2)$, so that a general tangent vector at $\pi(p)$ can be written as $a_{1} \frac{\partial}{\partial x^{1}}+a_{2} \frac{\partial}{\partial x^{2}}+a_{3} \frac{\partial}{\partial x^{3}}$. We calculate the matrix of the isomorphism $\left.\pi_{*}\right|_{p}$ mapping the coordinates $\alpha_{x}, \alpha_{y}, \alpha_{z}$, in $R_{p *}(\sigma, \gamma \sigma):=R_{p *}\left(\alpha_{x}\left(i \sigma_{x}, \gamma i \sigma_{x}\right)+\alpha_{y}\left(i \sigma_{y}, \gamma i \sigma_{y}\right)+\right.$ $\left.\alpha_{z}\left(i \sigma_{z}, \gamma i \sigma_{z}\right)\right) \in R_{p *} \mathcal{P}$ to $\left(a_{1}, a_{2}, a_{3}\right)$, (cf. (6) ). Denote this matrix by $\Pi_{j, l}:=\Pi_{j, l}(p)$ with $j=1,2,3$ and $l=x, y, z$. We have

$$
\Pi_{j, l}(p)=\left.\pi_{*}\right|_{p} R_{p *}\left(i \sigma_{l}, i \gamma \sigma_{l}\right) x^{j} .
$$

For the sake of illustration, let us calculate $\Pi_{1, x}(p)$. This is given by (recall $p$ is defined in (17))

$\Pi_{1, x}(p):=\pi_{p *} R_{p *}\left(i \sigma_{x}, i \gamma \sigma_{x}\right) x^{1}=R_{p *}\left(i \sigma_{x}, i \gamma \sigma_{x}\right)\left(x^{1} \circ \pi\right)=\left.\frac{d}{d t}\right|_{t=0} x^{1} \circ \pi\left(e^{i \sigma_{x} t}\left(\begin{array}{cc}x & y \\ -y^{*} & x^{*}\end{array}\right), e^{i \gamma \sigma_{x} t}\left(\begin{array}{cc}z & w \\ -w^{*} & w^{*}\end{array}\right)\right)$.

This simplifies because $x^{1} \circ \pi$ does not depend on the second factor. Therefore the $\Pi_{1, x}(p)$ entry is the derivative at $t=0$ of the real part of the $(1,1)$ entry of the matrix

$$
e^{i \sigma_{x} t}\left(\begin{array}{cc}
x & y \\
-y^{*} & x^{*}
\end{array}\right)=\left(\begin{array}{cc}
\cos (t) & i \sin (t) \\
i \sin (t) & \cos (t)
\end{array}\right)\left(\begin{array}{cc}
x & y \\
-y^{*} & x^{*}
\end{array}\right)
$$

This leads to the result

$$
\Pi_{1, x}(p)=-y_{I}
$$

The quantities

$$
\Delta_{1}:=z_{I} y_{R}-x_{I} w_{R}, \quad \Delta_{2}:=z_{I} y_{I}-x_{I} w_{I}, \quad \Delta_{3}:=w_{R} y_{I}-w_{I} y_{R},
$$

appear routinely in calculations that follow.

Similar calculations to the ones above for $\Pi_{1, x}(p)$ lead to the full matrix $\Pi(p)$, which is given by

$$
\Pi(p):=\left(\begin{array}{ccc}
-y_{I} & -y_{R} & -x_{I} \\
-\gamma w_{I} & -\gamma w_{R} & -\gamma z_{I} \\
(\gamma-1) \Delta_{1}+\gamma z_{R} y_{I}+w_{I} x_{R} & (1-\gamma) \Delta_{2}+w_{R} x_{R}+\gamma z_{R} y_{R} & (\gamma-1) \Delta_{3}+x_{R} z_{I}+\gamma z_{R} x_{I}
\end{array}\right)
$$


The determinant of this matrix is different from zero if and only if $p$ is in the regular part and it is another invariant under the action of $S U(2)$ on $S U(2) \times S U(2)$ (cf. Appendix B). It can be explicitly computed as

$$
\operatorname{det}(\Pi(p))=\gamma(\gamma-1)\left(\Delta_{1}^{2}+\Delta_{2}^{2}+\Delta_{3}^{2}\right),
$$

which can be seen to be equal to zero if and only if condition (16) is verified. The invariant $\Delta:=$ $\Delta_{1}^{2}+\Delta_{2}^{2}+\Delta_{3}^{2}$ can be expressed in terms of the (minimal) invariants $x_{R}, z_{R}$ and $x^{3}$ in 18 as

$$
\Delta=\Delta_{1}^{2}+\Delta_{2}^{2}+\Delta_{3}^{2}=\left(1-x_{R}^{2}\right)\left(1-z_{R}^{2}\right)-\left(x^{3}\right)^{2} .
$$

When we design a control law, the components $a_{1}, a_{2}, a_{3}$ of the tangent vector at every time $t$ in the tangent space at $\pi(p(t))$ are given by the derivatives $\dot{x}^{1}, \dot{x}^{2}, \dot{x}^{3}$ of the desired trajectory in the quotient space. The corresponding components, $\alpha_{x}, \alpha_{y}$ and $\alpha_{z}$, of the tangent vector in $R_{p(t) *} \mathcal{P}$ give the appropriate control functions $\left(u_{x}, u_{y}, u_{z}\right)$. The matrix $\Pi(p)$ in 22 gives the map from the control to trajectories. Since we want to specify trajectories and compute the corresponding controls, we need the inverse of the matrix $\Pi(p)$ (cf. (7)). This is found from $(22)$ to be

$$
\operatorname{det}(\Pi(p)) \Pi^{-1}(p):=\left(\begin{array}{ccc}
\gamma(\gamma-1)\left(-w_{R} \Delta_{3}-z_{I} \Delta_{2}\right)+\gamma^{2} z_{R} \Delta_{1} & (\gamma-1)\left(x_{I} \Delta_{2}+y_{R} \Delta_{3}\right)+x_{R} \Delta_{1} & \gamma \Delta_{1} \\
\gamma(\gamma-1)\left(w_{I} \Delta_{3}-z_{I} \Delta_{1}\right)-\gamma^{2} z_{R} \Delta_{2} & (\gamma-1)\left(x_{I} \Delta_{1}-y_{I} \Delta_{3}\right)-x_{R} \Delta_{2} & -\gamma \Delta_{2} \\
\gamma(\gamma-1)\left(w_{I} \Delta_{2}+w_{R} \Delta_{1}\right)+\gamma^{2} z_{R} \Delta_{3} & -(\gamma-1)\left(y_{I} \Delta_{2}+y_{R} \Delta_{1}\right)+x_{R} \Delta_{3} & \gamma \Delta_{3}
\end{array}\right) .
$$

We remark that $\Pi^{-1}(p)$ is not defined if we are in the singular part of the space $G=S U(2) \times S U(2)$ as the determinant of $\Pi$ is zero there. This is in particular true at the beginning as the initial point $p \in S U(2) \times S U(2)$ is the identity. In order to follow a prescribed trajectory in the quotient space $G_{r e g} / S U(2)$, we need therefore to apply a preliminary control to drive the state to an arbitrary point in $G_{r e g}$ and after that we shall apply the control corresponding to a prescribed trajectory in the quotient space.

The preliminary control in an interval $\left[0, T_{1}\right]$ to move the state from the singular part of the quotient space has to involve at least two different directions in the tangent space. In other terms, if we use $\sigma(t)=u(t) \sigma$ for some function $u=u(t)$ and a constant matrix $\sigma \in \mathfrak{s u}(2)$ we remain in the singular part. To see this, notice that if $d:=\int_{0}^{T_{1}} u(s) d s$, then the solution of 15 will be $\left(U_{f}, V_{f}\right)=\left(e^{d \sigma}, e^{\gamma d \sigma}\right)$, a pair that satisfies the condition (16). Therefore the simplest control strategy of moving in one direction only will not work if we want to move the state from the singular part. Furthermore, we want $u_{x}(0)=u_{y}(0)=$ $u_{z}(0)=0$ and $u_{x}\left(T_{1}\right)=u_{y}\left(T_{1}\right)=u_{z}\left(T_{1}\right)=0$ to avoid discontinuities at the initial time $t=0$ and at the time of concatenation with the second portion of the control. We propose to prescribe a trajectory for $U=U(t)$ in (15) and, from that trajectory, to derive the control to be used in the equation for $V=V(t)$ in (15). We choose a smooth function $\delta:=\delta(t)$ such that $\delta(0)=0$ and $\delta\left(T_{1}\right)=\delta_{0} \neq 0$, and $\dot{\delta}(0)=\dot{\delta}\left(T_{1}\right)=0$. We also choose a smooth function $\epsilon:=\epsilon(t)$, with $\epsilon(0)=\epsilon_{0} \neq 0$ and $\epsilon\left(T_{1}\right)=0$, and $\dot{\epsilon}(0)=\dot{\epsilon}\left(T_{1}\right)=0$. We choose for $U=U(t)$ in 15

$$
U(t)=\left(\begin{array}{cc}
\cos (\delta(t)) & e^{i \epsilon(t)} \sin (\delta(t)) \\
-e^{-i \epsilon(t)} \sin (\delta(t)) & \cos (\delta(t))
\end{array}\right)
$$

which at time $T_{1}$ gives

$$
U\left(T_{1}\right)=\left(\begin{array}{cc}
\cos \left(\delta_{0}\right) & \sin \left(\delta_{0}\right) \\
-\sin \left(\delta_{0}\right) & \cos \left(\delta_{0}\right)
\end{array}\right) .
$$

\footnotetext{
${ }^{7}$ This can be seen by expanding the left hand side using the definitions of $\Delta_{1,2,3} \sqrt{21}$ and the right hand side using the definition of $x^{3}$ 188, so that 24) reduces to $y_{I}^{2} w_{R}^{2}+w_{I}^{2} y_{R}^{2}+y_{R}^{2} z_{I}^{2}+x_{I}^{2} w_{R}^{2}+x_{I}^{2} w_{I}^{2}+y_{I}^{2} z_{I}^{2}=\left(1-x_{R}^{2}\right)\left(1-z_{R}^{2}\right)-z_{I}^{2} x_{I}^{2}-y_{R}^{2} w_{R}^{2}-y_{I}^{2} w_{I}^{2}$, and writing $\left(1-x_{R}^{2}\right)=x_{I}^{2}+y_{R}^{2}+y_{I}^{2}$ and $\left(1-z_{R}^{2}\right)=z_{I}^{2}+w_{R}^{2}+w_{I}^{2}$, we obtain an identity.
} 
The corresponding control $\sigma$ is $\sigma(t)=\dot{U} U^{\dagger}$, which is

$$
\sigma(t)=\left(\begin{array}{cc}
i \dot{\epsilon} \sin ^{2}(\delta) & \dot{\delta} e^{i \epsilon}+\frac{i}{2} \dot{\epsilon} \sin (2 \delta) e^{i \epsilon} \\
-\dot{\delta} e^{-i \epsilon}+\frac{i}{2} \dot{\epsilon} \sin (2 \delta) e^{-i \epsilon} & -i \dot{\epsilon} \sin ^{2}(\delta)
\end{array}\right) .
$$

Placing this in the second equation of (15) and integrating numerically we obtain the values for $V\left(T_{1}\right)$, the second component of $(U, V)$, and therefore the values of $\left(z_{R}, z_{I}, w_{R}, w_{I}\right)$. Using these values and the expression for $\left(x_{R}, x_{I}, z_{R}, z_{I}\right)$ in (27), and using the formula for $\Delta$ given in (24), we obtain

$$
\Delta=\Delta_{1}^{2}+\Delta_{2}^{2}+\Delta_{3}^{2}=\sin ^{2}\left(\delta_{0}\right)\left(1-z_{R}^{2}\left(T_{1}\right)-w_{R}^{2}\left(T_{1}\right)\right)=\sin ^{2}\left(\delta_{0}\right)\left(z_{I}^{2}\left(T_{1}\right)+w_{I}^{2}\left(T_{1}\right)\right)
$$

which has to be different from zero in order for the state to be in the regular part.

The second portion of the control depends on the trajectory followed, $\left(x^{1}, x^{2}, x^{3}\right)=\left(x^{1}(t), x^{2}(t), x^{3}(t)\right)$, and it is obtained by multiplying by $\Pi^{-1}$ in 25$)\left(\dot{x}^{1}, \dot{x}^{2}, \dot{x}^{3}\right)$. The trajectory $\left(x^{1}, x^{2}, x^{3}\right)$ is almost completely arbitrary. However it has to satisfy certain conditions which we now discuss. Let us denote the interval where the second part of the control is used by $\left[0, T_{2}\right]$. The initial condition $\left(x^{1}(0), x^{2}(0), x^{3}(0)\right)$ has to agree with the one given by the previous interval of control. The final condition $\left(x^{1}\left(T_{2}\right), x^{2}\left(T_{2}\right), x^{3}\left(T_{2}\right)\right)$ has to agree with the orbit of the desired final condition. Moreover, care has to be taken to make sure that the trajectory is such that $\Delta$ in $(24)$ is never zero because this would create a singularity in $\Pi^{-1}(p)$. Furthermore we need $\dot{x}^{1}(0)=\dot{x}^{2}(0)=\dot{x}^{3}(0)=0$, which gives $\sigma(0)=0$, to ensure continuity with the control in the previous interval, and we also choose $\dot{x}^{1}\left(T_{2}\right)=\dot{x}^{2}\left(T_{2}\right)=\dot{x}^{3}\left(T_{2}\right)=0$ to ensure that the control is switched off at the end of the procedure. Finally, the functions $\left(x^{1}, x^{2}, x^{3}\right)$ have to be representative of a possible trajectory for special unitary matrices. This means that, with $\vec{V}:=\left(x_{R}, x_{I}, y_{R}, y_{I}\right)^{T}$ and $\vec{W}:=\left(z_{R}, z_{I}, w_{R}, w_{I}\right)^{T},\|\vec{V}(t)\|^{2}=\|\vec{W}(t)\|^{2}=1$, at every $t$. Therefore $\left|x_{R}(t)\right|<1$ at every $t$, $\left|z_{R}(t)\right|<1$, at every $t$ (to avoid singularity), and from the Schwartz inequality $|\vec{V} \cdot \vec{W}| \leq 1$ we also must have $\left|x_{R} z_{R}+x^{3}\right| \leq 1$ and therefore

$$
-1-x_{R} z_{R} \leq x^{3} \leq 1-x_{R} z_{R}
$$

Once the functions $\left(\dot{x}^{1}, \dot{x}^{2}, \dot{x}^{3}\right)$ are chosen, the system to integrate numerically is 15$)$ with $\left(u_{x}, u_{y}, u_{z}\right)$ given by $\Pi^{-1}(p)\left(\dot{x}^{1}, \dot{x}^{2}, \dot{x}^{3}\right)^{T}$. By deriving $\left(u_{x}, u_{y}, u_{z}\right)$ using the explicit expression of $\Pi^{-1}$ given in (25) and replacing into (15), it is possible to obtain a simplified system of differential equations for $\left(x_{R}, x_{I}, \ldots, z_{R}, z_{I}, \ldots, w_{I}\right)$ without implementing the preliminary step of calculating the control. We found this system to be more stable in numerical integration with MATLAB and report it in Appendix A for future use.

\subsection{Numerical example: Driving to two different Hadamard gates}

We now apply the above technique to a specific numerical example: The problem is to drive the system (13) so that the first spin performs the Hadamard-type gate

$$
H_{1}:=\left(\begin{array}{cc}
\frac{1}{\sqrt{2}} & \frac{1}{\sqrt{2}} \\
\frac{-1}{\sqrt{2}} & \frac{1}{\sqrt{2}}
\end{array}\right)
$$

and the second spin performs the Hadamard gate

$$
H_{2}:=\left(\begin{array}{cc}
\frac{1}{\sqrt{2}} & \frac{-i}{\sqrt{2}} \\
\frac{-i}{\sqrt{2}} & \frac{1}{\sqrt{2}}
\end{array}\right)
$$


We want to drive system 15$)$ to $\left(U_{f}, V_{f}\right)=\left(H_{1}, H_{2}\right)$. The orbit of the desired final condition is characterized by the invariant coordinates

$$
x^{1}=x_{R}=\frac{1}{\sqrt{2}}, \quad x^{2}=z_{R}=\frac{1}{\sqrt{2}}, \quad x^{3}=x_{I} z_{I}+y_{R} w_{R}+y_{I} w_{I}=0 .
$$

We take a physical value for the ratio $\gamma$ between the two gyromagnetic ratios. In particular we will choose $\gamma \approx \frac{1}{0.2514}$ which corresponds to the Hydrogen-Carbon $\left({ }^{1} H-{ }^{13} C\right)$ system also considered in [10].

We first consider the control that moves the state away from the singular part, in a time interval $[0,1]$. We choose $\sigma$ in 28 with the functions $\delta$ and $\epsilon$ as follows:

$$
\delta=6 \delta_{0}\left(\frac{t^{2}}{2}-\frac{t^{3}}{3}\right), \quad \epsilon=\epsilon_{0}+6 \epsilon_{0}\left(\frac{t^{3}}{3}-\frac{t^{2}}{2}\right) .
$$

With these functions $\delta$ and $\epsilon, \sigma$ satisfies all the requirements described above. From (34) and (28) we obtain the controls $u_{x}, u_{y}, u_{z}$ which replaced into (15) give the dynamics in the interval $\left[0, T_{1}\right]=[0,1]$. Numerical integration with the values of the parameters $\delta_{0}=0.5$ and $\epsilon_{0}=1$, gives the following conditions at time $T_{1}=1$ (cf. (27)

$$
U(1)=\left(\begin{array}{cc}
\cos (0.5) & \sin (0.5) \\
-\sin (0.5) & \cos (0.5)
\end{array}\right), \quad V(1) \approx\left(\begin{array}{cc}
-0.3472+i 0.7769 & -0.5123-i 0.1157 \\
0.5123-i 0.1157 & -0.3472-i 0.7769
\end{array}\right)
$$

The value of $\Delta$ is, according to $29, \Delta \approx \sin ^{2}(0.5)\left((0.7769)^{2}+(0.1157)^{2}\right) \neq 0$, as desired.

The values of the variables to be used as initial conditions in the integration in the subsequent interval of the procedure are $x_{R}(1)=x^{1}(1)=\cos (0.5), \quad x_{I}(1)=0, \quad y_{R}(1)=\sin (0.5), \quad y_{I}(1)=$ $0, \quad z_{R}(1)=x^{2}(1)=-0.3472, \quad z_{I}(1)=0.7769, \quad w_{R}(1)=-0.5123, \quad w_{I}(1)=-0.1157$, and $x^{3}(1)=$ $x_{I}(1) z_{I}(1)+y_{R}(1) w_{R}(1)+y_{I}(1) w_{I}(1)=\sin (0.5) \times(-0.5123) \approx-0.2456$. For the subsequent interval $\left[0, T_{2}\right]$ we choose the trajectory $x^{1}(t), x^{2}(t), x^{3}(t)$ in the quotient space as follows: $T_{2}=10$ and the trajectory in the interval $\left[0, T_{2}\right]$ is

$$
\begin{gathered}
x^{1}(t)=-\frac{1}{500}\left(\frac{1}{\sqrt{2}}-\cos (0.5)\right) t^{3}+\frac{3}{100}\left(\frac{1}{\sqrt{2}}-\cos (0.5)\right) t^{2}+\cos (0.1) \\
x^{2}(t)=-\frac{1}{500}\left(\frac{1}{\sqrt{2}}+0.3472\right) t^{3}+\frac{3}{100}\left(\frac{1}{\sqrt{2}}+0.3472\right) t^{2}-0.3472 \\
x^{3}(t):=\frac{-0.2456}{500} t^{3}-\frac{3 \times(-0.2456)}{100} t^{2}-0.2456,
\end{gathered}
$$

which are easily seen to satisfy the conditions at the endpoints. Moreover by plotting $x^{1}$ and $x^{2}$ we see that $\left|x^{1}(t)\right| \leq 1$ and $\left|x^{2}(t)\right| \leq 1$ for every $t \in[0,10]$ (Figure 1). By plotting $x^{3}$ vs $1-x^{1} x^{2}$ and $-1-x^{1} x^{2}$ (Figure 22 we find that $-1-x^{1} x^{2}(t) \leq x^{3}(t) \leq 1-x^{1} x^{2}(t)$ for every $t \in[0,10]$ as required from condition (30). By plotting $\Delta=\Delta(t)$ in $[0,10]$ we know that $\Delta(t) \neq 0$ for every $t \in[0,10]$ (Figure 3). Therefore the whole trajectory is in the regular part.

The full trajectory, in the union of the two intervals, and with the concatenation of the two controls is depicted in Figure 4. Let us denote the full control by $\hat{\sigma}=\hat{\sigma}(t)=u_{x} i \sigma_{x}+u_{y} i \sigma_{y}+u_{z} i \sigma_{z}$. The final condition $\left(\hat{U}_{f}, \hat{V}_{f}\right)$ is given by

$$
\hat{U}_{f}=\left(\begin{array}{cc}
0.7071-0.2795 i & 0.5913+0.2685 i \\
-0.5913+0.2685 i & 0.7071+0.2795 i
\end{array}\right), \quad \hat{V}_{f}=\left(\begin{array}{cc}
0.7071+0.2708 i & 0.3718-0.5369 i \\
-0.3718-0.5369 i & 0.7071-0.2708 i
\end{array}\right) .
$$






Figure 1: Prescribed $x^{1}$ and $x^{2}$ variables in the (second) interval $[0,10]$.

This condition, as expected, is in the same orbit as the desired final condition $\left(H_{1}, H_{2}\right)$ in $(31),(32)$, that is, there exists a matrix $K \in S U(2)$ such that $K \hat{U}_{f} K^{\dagger}=H_{1}$ and $K \hat{V}_{f} K^{\dagger}=H_{2}$. The matrix $K$ solving these equations is found to be

$$
K=\left(\begin{array}{cc}
0.1485-0.2460 i & -0.2444+0.9260 i \\
0.2444+0.9260 i & 0.1485+0.2460 i
\end{array}\right) .
$$

In particular, to find $K$ one can diagonalize $\hat{U}_{f}$ and $H_{1}$, i.e., $\hat{U}_{f}=P \Lambda P^{\dagger}$ and $H_{1}=R \Lambda R^{\dagger}$ for a diagonal matrix $\Lambda$, so that, from $K P \Lambda P^{\dagger} K^{\dagger}=R \Lambda R^{\dagger}$, we find that $R^{\dagger} K P=D$, for $D$, a diagonal matrix. This matrix is found by solving $D P^{\dagger} \hat{V}_{f} P=R^{\dagger} H_{2} R D$. The control $K \hat{\sigma} K^{\dagger}$ steers then to the desired final condition. The resulting trajectory leading to the desired final condition (31), (32) is given in Figure 5 .

\section{References}

[1] F. Albertini and D. D'Alessandro, On symmetries in time optimal control, sub-Riemannian geometries and the K-P problem, Journal of Dynamical and Control Systems, (2018), Vol. 23, n.1.

[2] F. Albertini and D. D'Alessandro, Time optimal simultaneous control of two level quantum systems, Automatica, Vol. 74, December 2016, pp. 55-62.

[3] F. Albertini, D. D'Alessandro and B. Sheller, Sub-Riemannian geodesics on $S U(n) / S(U(n-1) \times$ $U(1)$ ) and optimal control of three level quantum systems, submitted to IEEE Transactions on Automatic Control, ArXiv: 1803.06687.

[4] C Altafini and F Ticozzi, Modeling and control of quantum systems: An introduction IEEE Transactions on Automatic Control, 57 (8), 1898-1917

[5] U. Boscain, T. Chambrion and J. P. Gauthier, On the K-P problem for a three level quantum system: Optimality implies resonance, Journal of Dynamical and Control Systems, Vol. 8, No. 4, October 2002, 547-572, (2002). 


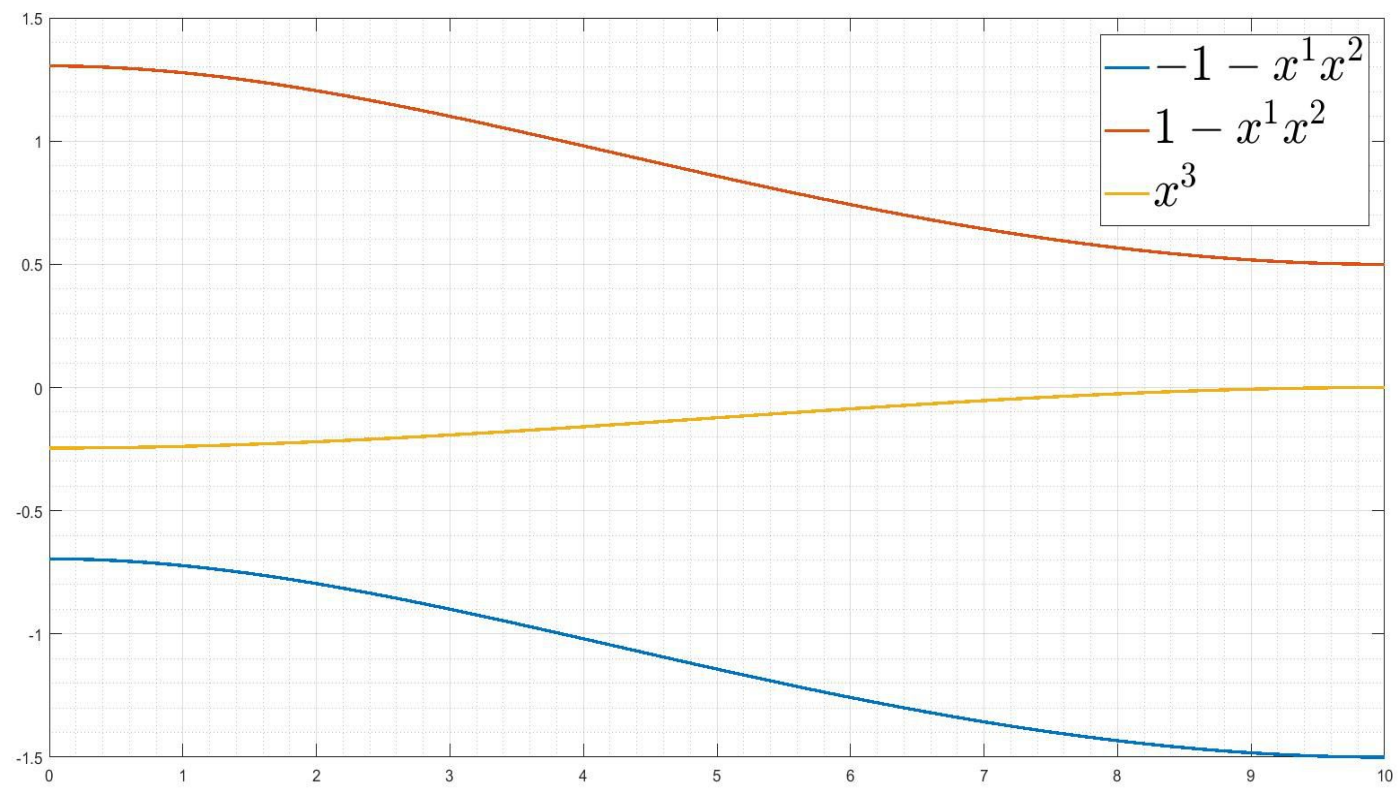

Figure 2: $x^{3}=x^{3}(t)$ vs $-1-x^{1}(t) x^{2}(t)$ and $1-x^{1}(t) x^{2}(t)$ in the (second) interval $[0,10]$.

[6] G. E. Bredon, Introduction to Compact Transformation Groups, Pure and Applied Mathematics, Vol. 46, Academic Press, New York, 1972.

[7] D. D'Alessandro, Introduction to Quantum Control and Dynamics, CRC Press, Boca Raton FL, August 2007.

[8] D. D'Alessandro and B. Sheller, On $K-P$ sub-Riemannian Problems and their Cut Locus, to appear in European Control Conference Proceedings 2019, different version available on https://arxiv.org starting April, 30, 2019.

[9] S. Glaser, U. Boscain, T. Calarco, C. Koch, W. Köckenberger, R. Kosloff, I. Kuprov, B. Luy, S. Schirmer, T. Schulte-Herbrggen, D. Sugny, F. Wilhelm Training Schrdinger's cat: quantum optimal control, Eur. Phys. J. D (2015) 69: 279.

[10] Y. Ji, J. Bian, M. Jiang, D. DAlessandro and X. Peng, Time-optimal control of independent spin- $1 / 2$ systems under simultaneous control, Physical Review A 98, 062108 December 2018.

[11] V. Jurdjević and H. Sussmann, Control systems on Lie groups, Journal of Differential Equations, 12, 313-329, (1972).

[12] S. Helgason, Differential geometry, Lie groups and symmetric spaces, Academic Press, New York, 1978.

[13] V Ramakrishna, K. L. Flores, H. Rabitz, and R. J. Ober, Quantum control by decompositions of SU(2), Phys. Rev. A 62, 053409 October, 13, 2000

[14] B. Sheller, Symmetry reduction in $K-P$ problems, Ph.D. Thesis, Department of Mathematics, Iowa State University, Spring 2019.

[15] V. O. Shkolnikov and G. Burkard, Effective Hamiltonian theory of the geometric evolution of quantum systems, https://arxiv.org/pdf/1810.00193.pdf 


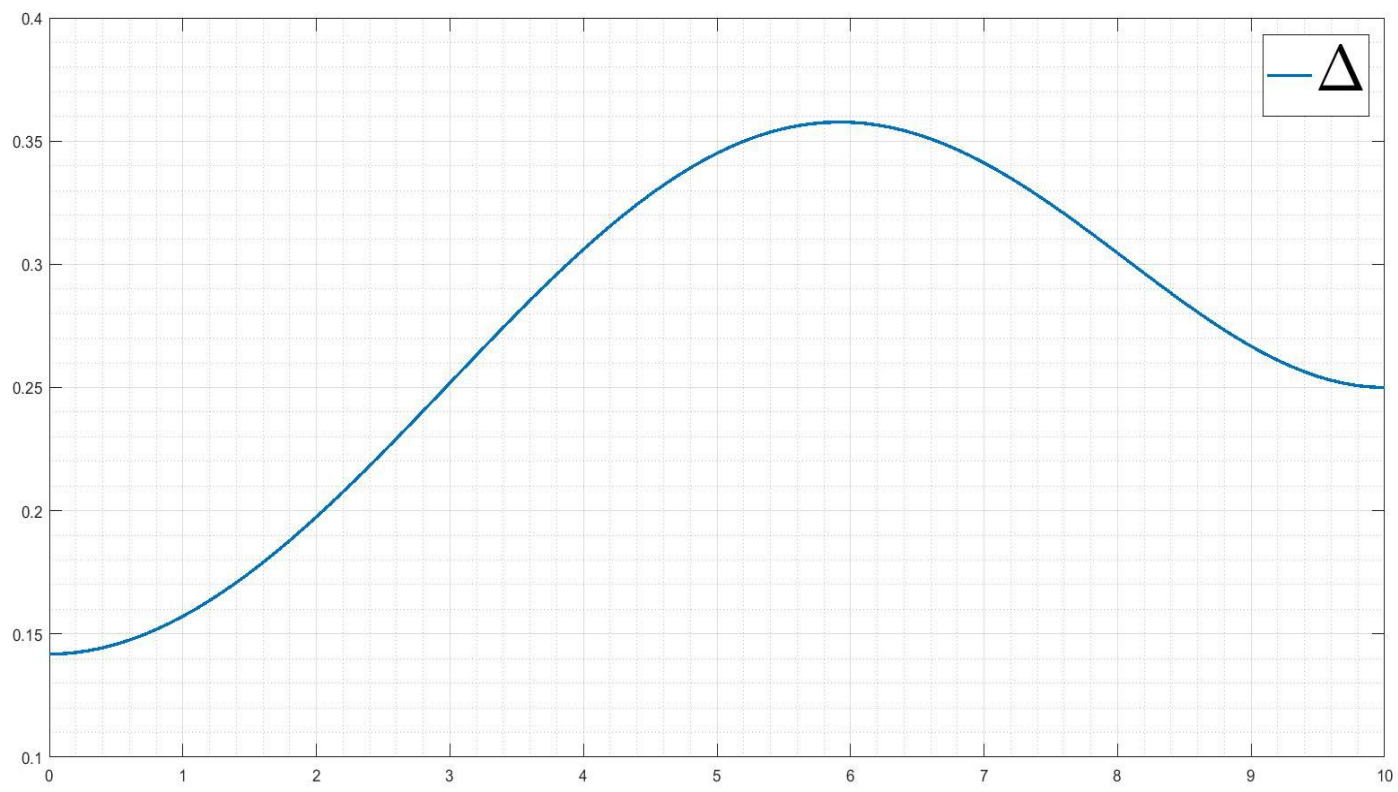

Figure 3: $\Delta=\Delta(t)=\Delta_{1}^{2}(t)+\Delta_{2}^{2}(t)+\Delta_{3}^{2}(t)$ in the (second) interval $[0,10]$.

\section{Appendix A: System of ODE's for the simultaneous control of two quantum bits in Subsection 3.4}

We derived the system of ODE's (15) with $\left(u_{x}, u_{y}, u_{z}\right)$ obtained from $\left(u_{x}, u_{y}, u_{z}\right)^{T}=\Pi^{-1}(p)\left(\dot{x}^{1}, \dot{x}^{2}, \dot{x}^{3}\right)^{T}$ with $\Pi^{-1}$ given in 25]. We define in the following $\Delta:=\Delta_{1}^{2}+\Delta_{2}^{2}+\Delta_{3}^{2}, Z:=x^{3}:=x_{I} z_{I}+y_{R} w_{R}+y_{I} w_{I}$. Simplifications are obtained using the following two relations which are directly verified.

$$
\begin{aligned}
& y_{I} \Delta_{1}-y_{R} \Delta_{2}+x_{I} \Delta_{3}=0, \\
& w_{I} \Delta_{1}-w_{R} \Delta_{2}+z_{I} \Delta_{3}=0 .
\end{aligned}
$$

The system becomes with $a:=\dot{x}^{1}, b:=\dot{x}^{2}, c:=\dot{x}^{3}$

$$
\begin{aligned}
& \dot{x}_{R}=a \\
& \gamma(\gamma-1) \Delta \dot{x}_{I}=(1-\gamma) \Delta_{3} b+\left(\gamma^{2} z_{R} a+\gamma c+\gamma b x_{R}\right)\left(x_{R} \Delta_{3}-y_{I} \Delta_{2}-y_{R} \Delta_{1}\right)+\gamma(\gamma-1) a\left(\Delta_{3} Z+x_{R}\left(w_{I} \Delta_{2}+w_{R} \Delta_{1}\right)\right) \\
& \gamma(\gamma-1) \Delta \dot{y}_{R}=(\gamma-1) \Delta_{2} b+\left(\gamma^{2} z_{R} a+\gamma c+\gamma b x_{R}\right)\left(x_{I} \Delta_{1}-x_{R} \Delta_{2}-y_{I} \Delta_{3}\right)+\gamma(\gamma-1) a\left(-\Delta_{2} Z+x_{R}\left(w_{I} \Delta_{3}-z_{I} \Delta_{1}\right)\right) \\
& \gamma(\gamma-1) \Delta \dot{y}_{I}=(1-\gamma) \Delta_{1} b+\left(\gamma^{2} z_{R} a+\gamma c+\gamma b x_{R}\right)\left(x_{R} \Delta_{1}+x_{I} \Delta_{2}+y_{R} \Delta_{3}\right)+\gamma(\gamma-1) a\left(\Delta_{1} Z-x_{R}\left(w_{R} \Delta_{3}+z_{I} \Delta_{2}\right)\right) \\
& \dot{z}_{R}=b \\
& (\gamma-1) \Delta \dot{z}_{I}=\gamma(\gamma-1) \Delta_{3} a+\left(\gamma z_{R} a+\gamma c+x_{R} b\right)\left(z_{R} \Delta_{3}-w_{R} \Delta_{1}-w_{I} \Delta_{2}\right)+(1-\gamma) b\left(\Delta_{3} Z+z_{R}\left(y_{R} \Delta_{1}+y_{I} \Delta_{2}\right)\right) \\
& (\gamma-1) \Delta \dot{w}_{R}=\gamma(1-\gamma) \Delta_{2} a+\left(\gamma z_{R} a+\gamma c+x_{R} b\right)\left(z_{I} \Delta_{1}-\Delta_{3} w_{I}-z_{R} \Delta_{2}\right)+(\gamma-1) b\left(\Delta_{2} Z+z_{R}\left(x_{I} \Delta_{1}-y_{I} \Delta_{3}\right)\right) \\
& (\gamma-1) \Delta \dot{w}_{I}=\gamma(\gamma-1) \Delta_{1} a+\left(\gamma z_{R} a+\gamma c+x_{R} b\right)\left(\Delta_{1} z_{R}+z_{I} \Delta_{2}+w_{R} \Delta_{3}\right)+(\gamma-1) b\left(-\Delta_{1} Z+z_{R}\left(y_{R} \Delta_{3}+x_{I} \Delta_{2}\right)\right)
\end{aligned}
$$

\section{Appendix B : Invariance of the determinant of $\pi_{*}$}

Let $\mathcal{L}$ be a semisimple Lie algebra with decomposition $\mathcal{L}=\mathcal{K} \oplus \mathcal{P}$ and $[\mathcal{K}, \mathcal{K}] \subseteq \mathcal{K}$ and $[\mathcal{K}, \mathcal{P}] \subseteq \mathcal{P}$, and consider the conjugacy action of $e^{\mathcal{K}}$ on $e^{\mathcal{L}}$. Consider the natural projection $\pi: e^{\mathcal{L}} \rightarrow e^{\mathcal{L}} / e^{\mathcal{K}}$ and $p \in e^{\mathcal{L}}$ a regular point so that 


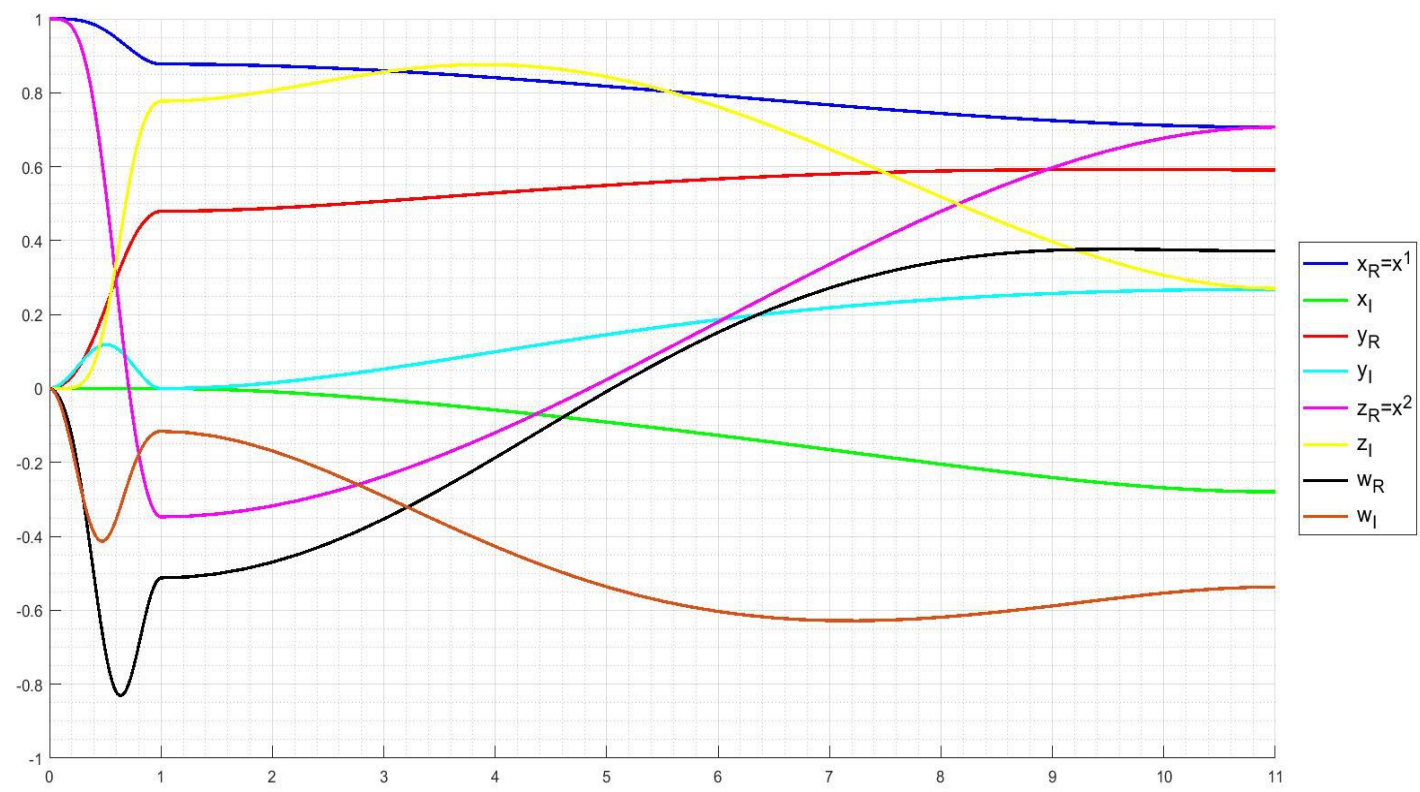

Figure 4: Trajectory under the preliminary control in the total interval $[0,11]$.

at $p, \pi_{*}$ is an isomorphism $\pi_{*}: R_{p *} \mathcal{P} \rightarrow T_{\pi(p)} e^{\mathcal{L}} / e^{\mathcal{L}}$. Given bases in $R_{p *} \mathcal{P}$ and $T_{\pi(p)} e^{\mathcal{L}} / e^{\mathcal{L}}$ the matrix $\Pi=\Pi(p)$ representing $\pi_{*}$ has determinant which is invariant under the action of $e^{\mathcal{K}}$, i.e., for every $k \in e^{\mathcal{K}}$

$$
\operatorname{det} \Pi(p)=\operatorname{det} \Pi\left(k p k^{-1}\right) .
$$

Proof. Let $\left\{B_{1}, \ldots, B_{m}\right\}$ be a basis of $\mathcal{P}$ so that $\left\{R_{p *} B_{1}, \ldots, R_{p *} B_{m}\right\}$ is a basis of $R_{p * \mathcal{P}}$. Let $x^{1}, \ldots, x^{m}$ be a set of coordinates for $e_{\text {reg }}^{\mathcal{L}} / e^{\mathcal{K}}$ in a neighborhood of $\pi(p)$. The $m \times m$ matrix $\Pi$ has entries

$$
\Pi_{j, l}(p)=\left(\pi_{*} R_{p *} B_{l}\right) x^{j},
$$

and it maps a vector $\left[\alpha^{1}, \ldots, \alpha^{m}\right]^{T}$ representing a tangent vector $V_{1} \in R_{p *} \mathcal{P}$, i.e., $V_{1}:=\sum_{l=1}^{m} \alpha^{l} R_{p *} B_{l}$ to a vector $\left[r^{1}, \ldots, r^{m}\right]^{T}$ representing a tangent vector $V_{2} \in T_{\pi(p)} e_{r e g}^{\mathcal{L}} / e^{\mathcal{K}}$, i. e., $V_{2}=\sum_{j=1}^{m} r^{j} \frac{\partial}{\partial x^{j}}$.

Let $P \in \mathcal{P}$ with $P:=\sum_{l=1}^{m} \alpha^{l} B_{l}$. Then, with $k \in e^{\mathcal{K}}$, we obtain 8

$$
\pi_{*} R_{p *} P=\pi_{*} R_{k p k^{-1}} k P k^{-1} .
$$

Therefore we have

$$
\begin{gathered}
\Pi_{j l}(p)=\left(\pi_{*} R_{p *} B_{l}\right) x^{j}=\left(\pi_{*} R_{k p k^{-1} *} k B_{l} k^{-1}\right) x^{j}= \\
\left(R_{k p k^{-1} *} k B_{l} k^{-1}\right) x^{j} \circ \pi=\left.\frac{d}{d t}\right|_{t=0} x^{j} \circ \pi\left(e^{k B_{l} k^{-1} t} k p k^{-1}\right) .
\end{gathered}
$$

Write $k B_{l} k^{-1}$ as $k B_{l} k^{-1}=\sum_{s=1}^{m} a_{l}^{s} B_{s}$, for an orthogonal matrix $9 a_{s}^{l}$. Therefore we have

$$
\begin{gathered}
\Pi_{j l}(p)=\left.\frac{d}{d t}\right|_{t=0} x^{j} \circ \pi\left(e^{k B_{l} k^{-1} t} k p k^{-1}\right)=\left.\frac{d}{d t}\right|_{t=0} x^{j} \circ \pi\left(e^{\sum_{s=1}^{m} a_{l}^{s} B_{s} t} k p k^{-1}\right)= \\
\left.\sum_{s=1}^{m} a_{l}^{s} \frac{d}{d t}\right|_{t=0}\left(x^{j} \circ \pi\left(e^{B_{s} t} k p k^{-1}\right)=\sum_{s=1}^{m} a_{l}^{s} \Pi_{j, s}\left(k p k^{-1}\right) .\right.
\end{gathered}
$$

\footnotetext{
${ }^{8}$ This follows from the definitions. For any function $f$, we have $\pi_{*} R_{p * P} f=R_{p *} P(f \circ \pi)=\left.\frac{d}{d t}\right|_{t=0} f \circ \pi\left(e^{P t} p\right)=\left.\frac{d}{d t}\right|_{t=0} f \circ$ $\pi\left(k e^{P t} p k^{-1}\right)=\left.\frac{d}{d t}\right|_{t=0} f \circ \pi\left(e^{k P k^{-1} t} k p k^{-1}\right)=\pi_{*} R_{k p k^{-1}} k P k^{-1}$.

${ }^{9}$ The fact that the matrix $\left\{a_{s}^{l}\right\}$, representing the adjoint action, is orthogonal is a consequence of the fact that the inner product, which is the Killing form on $\mathcal{P}$ is bi-invariant, and therefore it is not changed by the adjoint action.
} 


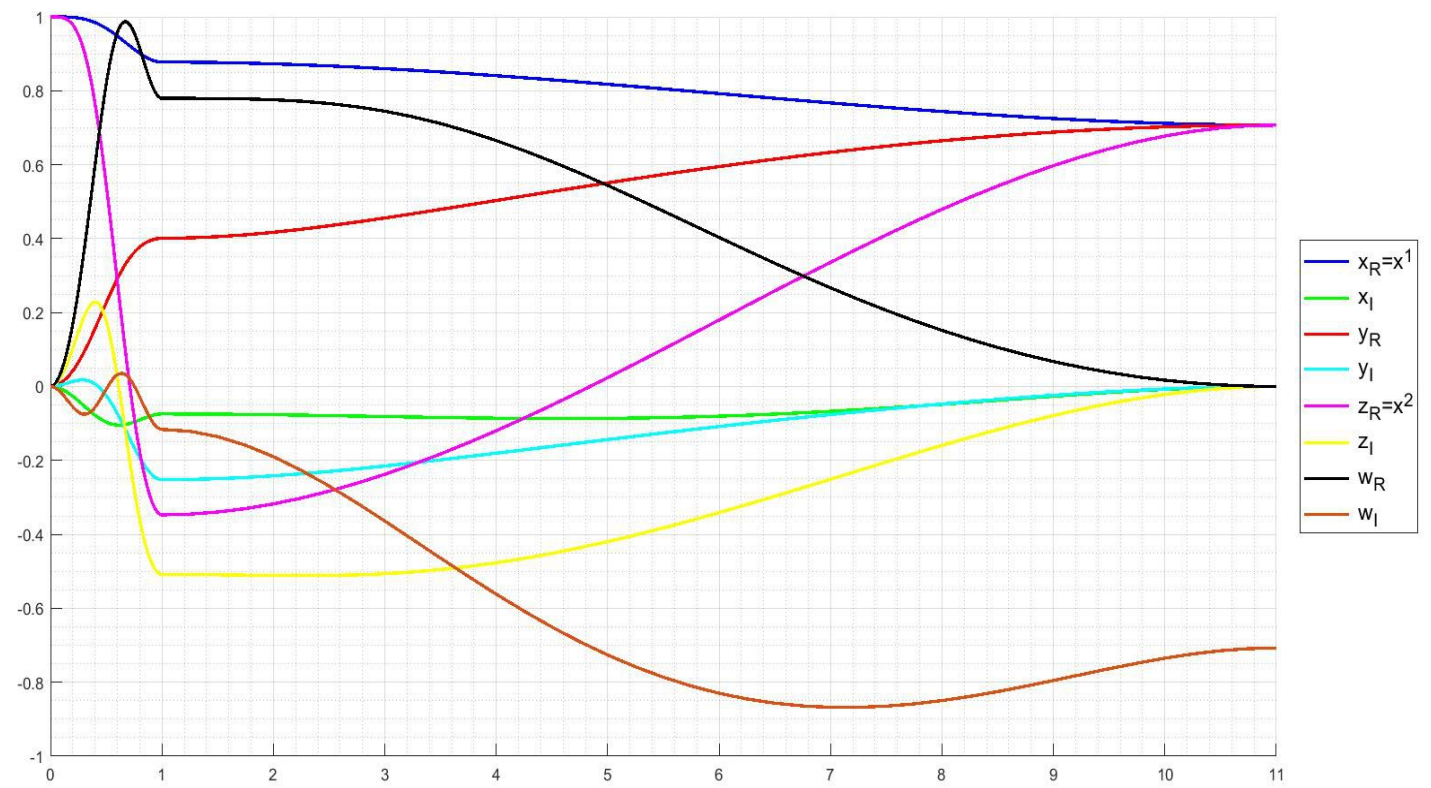

Figure 5: Trajectory under control algorithm leading to the desired final condition (31) and (32).

Therefore there exists an orthogonal matrix $A=A(k)$ so that

$$
\Pi(p)=\Pi\left(k p k^{-1}\right) A(k) .
$$

Taking the determinant of this relation and using $\operatorname{det}(A(k))=110$, we obtain $\operatorname{det}(\Pi(p))=\operatorname{det}\left(\Pi\left(k p k^{-1}\right)\right)$ as desired.

${ }^{10}$ Note that $\operatorname{det}(A(k)) \neq-1$, since $A: e^{\mathcal{K}} \rightarrow O(m, \mathbb{R}) ; A \mapsto A(k)$ is continuous and $\operatorname{det}(A(k))=\operatorname{det}\left(\mathbf{1}_{m}\right)=1$. 